\title{
An Improved Particle Swarm Optimization Algorithm Of Radial Basis Neural Network
}

\author{
Ji Weidong ${ }^{1,2}$, Sun Liping ${ }^{1}$, Wang Keqi ${ }^{1}$, Lv Liguo ${ }^{2}$ and Li Yue ${ }^{2}$ \\ ${ }^{1}$ College of Mechanical and Electrical Engineering, Northeast Forestry \\ University, Harbin, China \\ ${ }^{2}$ Computer science and Information Engineering College, Harbin Normal \\ University, Harbin, China \\ kingjwd@126.com
}

\begin{abstract}
The core issues of RBF network design are to design the minimum structure neural networks that can meet the accuracy requirements, in order to ensure the generalization ability of the network. For the purpose of simplifying the structure of RBF network, proposes a learning method of RBF network based on improved particle swarm. The method automatically constructs frugal structure of RBF network model by the combining algorithm of regularized least squares method and D- optimal experimental design; chooses three learning parameters of the combining algorithm that can affect network generalization performance by the improved particle swarm optimization algorithm. By nonlinear time series modeling, verifies the effectiveness of the method in this paper.
\end{abstract}

Keywords: RBF; particle swarm optimization; ROLS

\section{Introduction}

Radial Basis Function Neural Network is a commonly used three layer feed forward neural network. Compared with BP network, RBF network not only has a physiological basis, and the structure is simpler, faster learning speed. It not only has a solid theoretical foundation, a concise mathematical form, intuitive geometric interpretation, but also can solve the small sample, nonlinear, dimension and local minima, so it is widely used in the practical application and theory research [1-6]. It is a kind of multilayer feed forward network [7-11].

In the learning algorithm of RBF network, Chen put forward the method of combining ROLS with D-opt is in the RBF network structure design is one of the effective ways [12],the method by introducing D- optimal cost in cost function, not only to design a RBF network structure of thrift, but also can further improve the performance of the network model and robustness. Algorithm based on regularization technique to avoid network over fitting noise data to improve the generalization performance of the network, the regularization coefficient by Bayesian iterative learning method, but produced by the Bayesian iterative regularization coefficient is not optimal, it is only locally optimal under certain conditions [13]. This paper presents a RBF neural network learning method based on improved particle swarm optimization algorithm, the method proposed by ROLS combined with D-opt algorithm to automatically design RBF network structure of thrift, through the PSO global optimization to influence the three learning parameters of the generalization performance of RBF network: the regularization coefficient, base width parameter and D- optimal weights, and then the RBF network design has good generalization performance and the structure is very thrifty. 


\section{Combined with the Regularized Orthogonal Least Squares Method and D- Optimal Experiment Design Method}

In 2003 Chen, Hong and Harris for the ROLS algorithm and the D- optimal experiment design method are combined, by introducing D- optimal cost in cost function, not only can automatically design the structure of managing RBF network, and further improves the selected regression model performance and robustness. The specific algorithm sees literature ${ }^{[12]}$. D-optimal cost into the new cost function can further improve the model performance and robustness; and can automatic end the selection process regression factor, avoiding the error $\xi$ parameter settings. Coefficient regular $\lambda$ in the algorithm by Bayesian method to estimate learning and produce, but the iterative gradient method and the value of learning $\lambda$ is the current conditions (specific basis width parameter $\rho$ ) a local optimum found, is not the global optimal; On the other hand, base width parameter $\rho$ adjusting range in the network hidden nodes in the Gauss's function of radial function, so the generalization performance of the network with his choice has a very direct relationship; In addition to the D-optimal weight coefficient $\beta$ value is different, different regression factor will make the network selection in the regression process, which has a certain influence on the generalization performance of the final design network. In fact, the generalization ability of neural network with three parameters is formed between the super dimension complicated multi peak curve space, so we have to find a optimization algorithm with global searching ability, in its search space in an optimal global optimal $\lambda, \rho$ and $\beta$ parameters, so that the generalization performance of the network in the best state.

\section{IPSO}

In the particle swarm algorithm updates throughout the iterative process, the global extremum plays an important role, directly affects the quality of optimal solution, after the iteration, the global extremumis often located in the most advantages or near the optimum point. Objective to clone variation and update strategies designed in this paper is used to change the global extremum position vector in the population, to avoid falling into local extremum, improve the particle's fitness, so that the global minimum as far as possible at the advantages or near it. A mutation probability is $p_{p m}$, the general value of 0.001-0.1.

Component clone mutation operators on the global extremum position vector and the last random value ${ }^{[14]}$, clone mutation operator as the formula (1) design:

$$
\operatorname{gbest}(t)=\left\{\begin{array}{l}
\text { gbest }^{\prime}(t)+\Delta\left(t, \text { gbest }_{\max }-\right. \\
\text { gbest } \left.^{\prime}(t)\right) \quad \text { if } U(0,1) \leq 0.5 \\
\text { gbest }^{\prime}(t)-\Delta\left(t, \text { gbest }^{\prime}(t)-\right. \\
\text { gbest } \left._{\min }\right) \quad \text { else }
\end{array}\right.
$$

Here, $g b e s t^{\prime}(t)$ is a clone of $g b e s t(t)$ value after.

$$
\Delta(t, d)=d\left(1-r^{\left(1-t / n_{t}\right)^{5}}\right)
$$

In type (2), $r$ a refers to the uniform distribution on the interval $(0,1), n_{t}$ is the maximum number of iterations.

Characteristics of the non-uniform mutation operator is $\Delta(t, d)$ returns a $[0, d]$ value in the range, and $\lim _{t \rightarrow+\infty} \Delta(t, d)=0$,to ensure that the mutation step size decreased with 
the time increase in $\mathrm{C}$, consistent with the spatial variation in the initial stage of evolution is relatively large, is also in line with the small space in the late evolutionary variation.

Particle swarm optimization, in each iteration, clone populations of global extreme $\operatorname{gbest}(t)$, forming a scale and nature are the same with the original $g b e s t(t)$ clone gbest $^{\prime}(t)$, clone mutation operation on the particle, the next step calculation clone particle's fitness, if higher than itself particle with itself particles were replaced, otherwise the position of the particle. Through clonal variation, population in the global extremum has self-learning ability, can make the particles enhance the ability to escape from local minimum, and improve the efficiency of algorithm.

\section{The Improved PSO RBF Method}

\subsection{The Basic Idea of RBF Network Design Method}

Shown in Figure 1 using the RBF network learning method structure diagram. The basic idea is using the improved PSO candidate solution $\lambda, \rho$ and $\beta$ parameters to find a set of optimal set, the definition of the fitness function value is minimum, by ROLS and D- optimal test algorithm (ROLS+D-opt) for automatic design of RBF network corresponding to the particle.

As shown in Figure 1, the improved PSO algorithm with real number coding, the $p$ particles in the solution space (candidate), according to the defined fitness function, each particle calculation of particle fitness value $f_{i}$, the population after several updates, iteration, the global best particle finally get is the best we need solution, and then by the RBF network ROLS+D-opt design structure of thrift.



Figure 1. Structure Diagram of RBF Network Learning Method

\subsection{Improved RBF Neural Network PSO Optimal Learning Method Based on Adaptive Selection Function}

In the RBF network design process, first of all will need data sets were divided into training set and test set, the known particles in the particle swarm algorithm in value, we divided the training sample set of automatic generation of RBF network by ROLS+D-opt, and then calculate the MSE test set, using MSE as the particle swarm algorithm's fitness function value.

With the actual output test sample set for $\hat{y}(x(k))$, the expected output is $y(x(k))$, and then the fitness function can be defined as a type (3). 


$$
\min f(K)=\frac{1}{n_{c}} \sum_{k=1}^{n_{c}}(y(x(k))-\widehat{y}(x(k)))^{2}
$$

$n_{c}$ as the test sample, find the best parameter combination $\mathrm{K}$ in search space by using improved PSO method, is $\left[\lambda_{i}, \rho_{i}, \beta_{i}\right]$, the definition of the fitness function value of the minimum.

\section{Experimental Examples}

Experiment on nonlinear time series modeling example, describes the RBF network learning method in this paper is very effective, and with some other designing method of RBF network are compared with experimental results.

Consider as the formula ( 4 ) for nonlinear time series ${ }^{[12]}$

$$
\begin{aligned}
& y(k)=\left(0.8-0.5 \exp \left(-y^{2}(k-1)\right)\right) y(k-1)- \\
& \left(0.3+0.9 \exp \left(-y^{2}(k-1)\right)\right) \cdot y(k-2)+ \\
& 0.1 \sin (\pi y(k-1))+e(k)
\end{aligned}
$$

The mean of $e(k)$ was 0 , the variance is 0.09 Gauss noise; the initial state is $y(0)=y(-1)=0.1$, and the resulting 1000 band noise sample data, taking the first 500 data are used to train the RBF network, after the 500 data are used to verify the performance of network. Not with the noise of the time series model is given by formula (5).

$$
\begin{gathered}
y_{d}(k)=\left(0.8-0.5 \exp \left(-y_{d}^{2}(k-1)\right)\right) y_{d}(k-1)- \\
\left(0.3+0.9 \exp \left(-y_{d}^{2}(k-1)\right)\right) \cdot y_{d}(k-2)+ \\
0.1 \sin \left(\pi y_{d}(k-1)\right)
\end{gathered}
$$

The noise of the 500 training data for training the RBF network model, the input of $x(k)=[y(k-1), y(k-2)]^{T}$ network, the output corresponding to the sequence in the T predicted value is $\hat{y}(k)$, with noise data to construct such as type (6) RBF network model.

$$
\widehat{y}(k)=\widehat{f}_{R B F}(x(k))
$$

$$
x(k)=[y(k-1), y(k-2)]^{T}
$$

Optimization of ROLS+D-Opt algorithm by PSO $\lambda, \rho$ and $\beta$ values of three parameters, parameters of $\lambda, \rho$ and $\beta$ search interval for $[1 e-6,1],[0.01,1.2]$ and $[1 e-6,1]$, the particle group number $\mathrm{N}=30$, mutation probability $p_{p m}=0.02$, the 30 generation of evolutionary computation, the best particle $\left[\lambda_{\text {opt }}, \rho_{\text {opt }}, \beta_{\text {opt }}\right]=[0.00015,1.1026,0.0901]$, corresponding to the fitness function value $f_{\text {opt }}=0.0921$.The fitness curves as shown in Figure 2. 




Figure 2. Fitness Value Changing Curve

Table 1 gives three methods to design RBF network respective experimental results. OLS+D-Opt algorithm based on the width parameter $\rho=1$, D- optimal weight coefficient $\beta=0.001$; ROLS algorithm for network base width $\rho=1$, produced by the Bayesian regularization coefficient, the allowable error of $\xi=1 e-6$ parameters; this algorithm is optimized for PSO.

Seen from Table 1, the learning algorithm and the OLS+D-Opt algorithm and ROLS algorithm performance is better than them. In general, the learning algorithm not only improves the learning accuracy and generalization capability, but also the RBF network structure design of thrift in the OLS+D-Opt algorithm and ROLS algorithm.

Table 1. Performance Comparison of Nonlinear Time Series Designed With Different Methods

\begin{tabular}{|c|c|c|c|}
\hline $\begin{array}{c}\text { Learning } \\
\text { method }\end{array}$ & $\begin{array}{c}\text { The } \\
\text { learning } \\
\text { erro } \\
\text { r }\end{array}$ & $\begin{array}{c}\text { The } \\
\text { prediction } \\
\text { error }\end{array}$ & $\begin{array}{c}\text { The } \\
\text { number } \\
\text { of hidden } \\
\text { nodes }\end{array}$ \\
\hline OLS+D-opt & $\begin{array}{c}0.10 \\
16\end{array}$ & 0.1019 & 37 \\
\hline ROLS & $\begin{array}{c}0.09 \\
54\end{array}$ & 0.0975 & 25 \\
\hline This paper & $\begin{array}{c}0.07 \\
90\end{array}$ & 0.0921 & 15 \\
\hline
\end{tabular}

\section{Conclusion}

This paper proposes a learning method of improved RBF neural network based on particle swarm optimization. The regularized orthogonal least squares method and Doptimal experiment design, algorithm can automatically construct RBF network model structure of thrift. RBF network structure, network structure designed by this method is more frugal than OLS+D-opt, ROLS designed, and generalization performance of the method is better. Through the modeling problem of nonlinear time series is verified. 


\section{Acknowledgment}

It is supported by Key Laboratory of inetlligent education and information engineering project in Heilongiiang province,the special funds innovative talents of science and technology research project in Harbin 2015(2015RAQXJ040), Heilongjiang province key discipline of computer aoolication technology(081203), Harbin Normal University 2016 annual practice innovation team, College students' innovative entrepreneurial training project in Heilongjiang province(201610231029).

\section{References}

[1] M. Song, D. Tao and X. Huang, "Three-Dimensional Face Reconstruction From a Single Image by a Coupled RBF Network", IEEE Transactions on Image Processing, vol. 21, no. 5, (2012), pp. 2887-2897.

[2] K. Meng, Z. Y. Dong and D. H. Wang, "A Self-Adaptive RBF Neural Network Classifier for Transformer Fault Analysis", IEEE Transactions on Power Systems, vol. 25, no. 3, (2010), pp. 13501360 .

[3] M. Hou and X. Han, "Constructive Approximation to Multivariate Function by Decay RBF Neural Network", IEEE Transactions on Neural Networks, vol. 21, no. 9, (2010), pp. 1517-1523.

[4] X. Hong and C. J. Harris, "Nonlinear Model Structure Design and Construction Using Orthogonal Least Squares and D-Optimality Design", IEEE Transactions on Neural Networks, vol. 13, no. 5, (2002), pp. $1245-1250$.

[5] W.-M. Qiu, D.-W. Guo, C. -Y. Zhang, Y.-X. Xu and G. Wang, "A Smart Grid Client-Side Testing Platform For Monitoring”, Mathematical Modelling Of Engineering Problems, vol.2, no.3, (2015), pp.17-20.

[6] Y. Yan, "A Practice Guide Of Predicting Resource Consumption In A Web Server", Review Of Computer Engineering Studies, vol.2, no.3, (2015), pp.1-8.

[7] H.-G. Han and J.-F. Qiao, "Adaptive Computation Algorithm for RBF Neural Network", IEEE Transactions on Neural Networks and Learning Systems, vol. 23, no. 2, (2012), pp. 342-347.

[8] W.E. Wong, V. Debroy, R. Golden, X. Xu and B. Thuraisingham, "Effective Software Fault Localization Using an RBF Neural Network", IEEE Transactions on Reliability, vol. 61, no. 1, (2012), pp. $149-169$.

[9] W. Yao, X. Chen, Y. Zhao and M. Tooren, "Concurrent Subspace Width Optimization Method for RBF Neural Network Modeling", IEEE Transactions on Neural Networks and Learning Systems, vol. 23, no. 2, (2012), pp. 247-259.

[10] Long Zhang, Kang Li, Er-Wei Bai. A New Extension of Newton Algorithm for Nonlinear System Modelling Using RBF Neural Networks", IEEE Transactions on Automatic Control, vol. 58, no. 11, (2013), pp. 2929-2933.

[11] L. Chen and J. Fang, "A Hybrid Prediction Method for Bridging GPS Outages in High-Precision POS Application”, IEEE Transactions on Instrumentation and Measurement, vol. 63, no. 6, (2014), pp. 16561665.

[12] S. Chen, X. Hong and C. J. Harris, "Sparse Kernel Regression Modeling Using Combined Locally Regularized Orthogonal Least Squares and D-Optimality Experimental Design", IEEE Transactions on Automatic Control, vol. 48, no. 6, (2003), pp. 1029-1036.

[13] S. Chen, Y. Wu and B. L. Luk, "Combined Genetic Algorithm Optimization and Regularized Orthogonal Least Squares Learning for Radial Basis Function Networks", IEEE Transactions on Neural Networks, vol. 10, no. 5, (1999), pp. 1239-1243.

[14] S.C. Esquivel and C. A. Coello, "the Use of Particle Swarm Optimization with Multimodal Functions", In Proceedings of the IEEE Transactions on Evotutionary Computation, vol. 2, (2003, pp. 1130-1136).

\section{Authors}

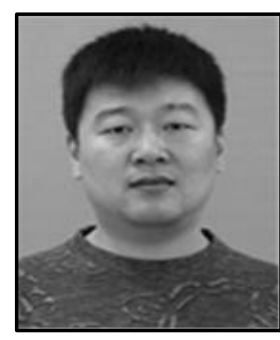

Ji Weidong, he received his M.Sc. in Control Theory and Control Engineering (2004), and Ph D in Mechanical Design and Theory (2013) from Northeast Forestry University. $\mathrm{He}$ is an associate Professor of Computer Science and Information Engineering. Since 2013 he is a Master Instructor. His current research interests include different aspects of Neural Network and Swarm Intelligence. 


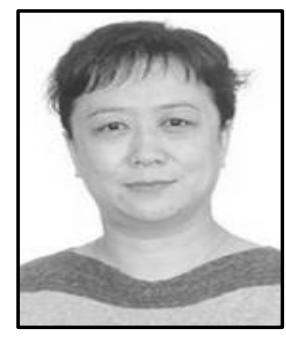

Sun Liping, she is a doctoral supervisor, control theory and control engineering, Northeast Forestry University.

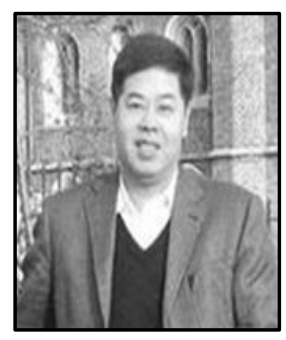

Wang Keqi, he is a doctoral supervisor, Northeast Forestry University, forestry engineering automation discipline leader. 
International Journal of Control and Automation

Vol. 9, No. 10 (2016) 